\title{
Coping with the COVID-19 crisis in Sub-Saharan Africa: let us not leave older people behind!
}

\author{
Moustapha Dramé $^{1,2} \cdot$ Lidvine Godaert $^{3} \cdot$ Kuate Tegueu Callixte ${ }^{4} \cdot$ Fiona Ecarnot $^{5} \cdot$ Nadine Simo-Tabue $^{6}$. \\ Maturin Tabue Teguo $6,7,8$
}

Received: 5 April 2020 / Accepted: 13 April 2020 / Published online: 22 April 2020

(c) European Geriatric Medicine Society 2020

Keywords COVID-19 $\cdot$ Older people $\cdot$ Sub-Saharan Africa

China's experience with the COVID-19 epidemic has taught us that the highest lethality rate is among older individuals and the time from onset of symptoms to death is shorter in those aged 70 years and older [1].

Armed with the knowledge coming out of the Chinese epidemic, high-resource countries rapidly implemented drastic measures among the older, and by definition more fragile, populations to halt transmission of disease, complications in cascade, loss of autonomy and ultimately, death [2]. This has important repercussions for the development of public health and clinical responses to the crisis [3].

Moustapha Dramé, Kuate Tegueu Callixte, Nadine Simo-

Tabue and Maturin Tabue Teguo are from Sub-Saharan African countries.

Moustapha Dramé

moustapha.drame@chu-martinique.fr

1 Faculty of Medicine, University of the French West Indies, Fort-de-France, Martinique

2 Department of Clinical Research and Innovation, University Hospitals of Martinique, Pierre Zobda-Quitman Hospital, CS 90632, 97261 Fort-de-France, Martinique

3 Department of Geriatrics, General Hospital of Valenciennes, Valenciennes, France

4 Department of Internal Medicine and Specialties, Faculty of Medicine and Biomedical Sciences, University of Yaoundé I, Yaoundé, Cameroon

5 Department of Cardiology, University Hospital Besancon and EA3920, University of Franche-Comté, Besancon, France

6 Department of Geriatrics, University Hospitals of Guadeloupe, Pointe-à-Pitre, Guadeloupe

7 LAMIA Research Team, University of the French West Indies, Pointe-à-Pitre, Guadeloupe

8 U1219 INSERM, University of Bordeaux, Bordeaux, France
At present, certain colleagues affirm that Africa is more prepared than ever to face the COVID-19 pandemic, thanks to the lessons they have learned from epidemics such as Ebola [4]. While we are fully confident that this is the case, it is nonetheless clear that in previous epidemics, as well as in the ongoing COVID-19 pandemic, older persons are, once again, paying the heaviest toll. Indeed, in the case of the current COVID-19 crisis, in Sub-Saharan Africa, no specific measures have been taken to protect elders, as there have been in high-income countries. In African countries, the main measures taken to stem the spread of the disease include closing borders, grounding commercial flights from severely affected countries, and postponing or cancelling major cultural or sporting events. These measures, while necessary and effective (on condition that they are properly implemented and respected), actually barely affect older people, who travel less than their younger counterparts and less frequently participate in large cultural or sports-related gatherings. Conversely, older people have a leading role in collective activities of daily life, which continue unimpeded, such as prayer groups, weddings, christenings and funerals. Older people also continue to stand in for parents, minding children with whom they are naturally in very close contact. All these circumstances combine to put older people at risk of contracting a range of communicable diseases, not least COVID-19.

If the epidemic continues along current trajectories, African countries are or will be among the last to be affected by the COVID-19 pandemic. Yet they are likely to draw but little benefit from the experience acquired in western countries, notably because the measures applied in high-income countries are not appropriate solutions for Sub-Saharan Africa. At present, total lockdown is the only measure that is unanimously considered to be efficacious. It has been widely implemented in western countries. Currently, the focal point 
of the epidemic, while we await, is either an efficacious treatment or a vaccine, either (or both) of which may take several months to emerge. The social, economic and cultural context in Sub-Saharan Africa renders total confinement virtually impossible. We also must not ignore the deleterious effect that lockdown has on social terms, isolating frail individuals and leaving them without support or resources.

In a context of poor medical infrastructures and weakened health systems, the governments of Sub-Saharan Africa do not appear to be fully cognizant of the risk that the older population is incurring. We are aware that in today's world, where social intermingling and movement is highly prevalent, it is difficult to implement targeted measures. However, giving the issue sufficient thought is a prerequisite to finding acceptable and efficacious solutions. Nkengasong and Mankoula [5] exhort us to act collectively and fast. We say hear, hear-but let us not leave the older population behind when we move forward! The COVID-19 crisis is afflicting the whole world and so the search for solutions should also include the whole world, taking account of local context. Similarly, provision of resources to fight the epidemic should also occur on a worldwide scale. Indeed, the crisis will only come to an end when every country in the world has vanquished COVID-19. In this regard, and to avoid any ageism, we applaud and adhere to the suggestion of Lloyd-Sherlock et al. [3] to take age explicitly into account in the development of national and global planning for COVID-19, and to convene a global expert group for older people to provide guidance and inform our response to this urgent global health threat.

Acknowledgements We would like to thank Professor Emeritus JeanPierre Michel for his very pertinent and sound advice.

Author contributions MD: literature search, study design, data collection, analysis and/or interpretation and manuscript writing; LG: study design, analysis and/or interpretation and manuscript revision; KTC: study design, analysis and/or interpretation and manuscript revision; FE: study design, analysis and/or interpretation and manuscript revision; NST: study design, analysis and/or interpretation and manuscript revision; MT: study design, analysis and/or interpretation and manuscript writing.

Funding There are no funding sources for this work.

\section{Compliance with ethical standards}

Conflict of interest There are no conflicts of interest to declare. Indeed, authors do not declare any financial interests or connections, direct or indirect, or other situations that might raise the question of bias in the work reported or the conclusions, implications, or opinions stated-including pertinent commercial or other sources of funding for the individual author(s) or for the associated department(s) or organization(s), personal relationships, or direct academic competition.

Ethical approval Not applicable.

Informed consent Not applicable.

\section{References}

1. Wang W, Tang J, Wei F (2020) Updated understanding of the outbreak of 2019 novel coronavirus (2019-nCoV) in Wuhan, China. J Med Virol 92(4):441-447

2. Garnier-Crussard A, Forestier E, Gilbert T, Krolak-Salmon P (2020) Novel coronavirus (COVID-19) epidemic: what are the risks for older patients? J Am Geriatr Soc. https://doi.org/10.1111/ jgs.16407 [Epub ahead of print]

3. Lloyd-Sherlock P, Ebrahim S, Geffen L, McKee M (2020) Bearing the brunt of COVID-19: older people in low and middle income countries. BMJ Clin Res Edn 368:m1052

4. Kapata N, Ihekweazu C, Ntoumi F et al (2020) Is Africa prepared for tackling the COVID-19 (SARS-CoV-2) epidemic. Lessons from past outbreaks, ongoing pan-African public health efforts, and implications for the future. Int J Infect Dis 93:233-236

5. Nkengasong JN, Mankoula W (2020) Looming threat of COVID-19 infection in Africa: act collectively, and fast. Lancet 395(10227):841-842

Publisher's Note Springer Nature remains neutral with regard to jurisdictional claims in published maps and institutional affiliations. 